\title{
Interactive visualization of public health indicators to support policymaking: An exploratory study
}

\author{
Moutasem Zakkar ${ }^{1,2}$, Kamran Sedig ${ }^{1}$ \\ 1. Insight Lab, Western University, Canada \\ 2. School of Public Health and Health Systems, University of Waterloo, Canada \\ * Moutasem Zakkar, School of Public Health and Health Systems, University of Waterloo, Canada. \\ mzakkar@uwaterloo.ca
}

\begin{abstract}
Purpose: The purpose of this study is to examine the use of interactive visualizations to represent data/information related to social determinants of health and public health indicators, and to investigate the benefits of such visualizations for health policymaking. Methods: The study developed a prototype for an online interactive visualization tool that represents the social determinants of health. The study participants explored and used the tool. The tool was evaluated using the informal user experience evaluation method. This method involves the prospective users of a tool to use and play with it and their feedback to be collected through interviews. Results: Using visualizations to represent and interact with health indicators has advantages over traditional representation techniques that do not allow users to interact with the information. Communicating healthcare indicators to policymakers is a complex task because of the complexity of the indicators, diversity of audiences, and different audience needs. This complexity can lead to information misinterpretation, which occurs when users of the health data ignore or do not know why, where, and how the data has been produced, or where and how it can be used. Conclusions: Public health policymaking is a complex process, and data is only one element among others needed in this complex process. Researchers and healthcare organizations should conduct a strategic evaluation to assess the usability of interactive visualizations and decision support tools before investing in these tools. Such evaluation should take into consideration the cost, ease of use, learnability, and efficiency of those tools, and the factors that influence policymaking.
\end{abstract}

Keywords: Interactive visualizations, social determinants of health, public health indicators, public health policymaking, exploratory study, informal user experience evaluation

Correspondence: mzakkar@uwaterloo.ca, kamrans@uwo.ca

DOI: 10.5210/ojphi.v9i2.8000

Copyright @2017 the author(s)

This is an Open Access article. Authors own copyright of their articles appearing in the Online Journal of Public Health Informatics. Readers may copy articles without permission of the copyright owner(s), as long as the author and OJPHI are acknowledged in the copy and the copy is used for educational, not-for-profit purposes 


\section{Introduction}

Health policymaking is a complex process that aims to control the functions of the health system, including health service organization, financing, payment, and health promotion [1]. To take appropriate actions, health policymakers require various kinds of information about the health system performance and public health problems and needs. There are many sources for this information, including medical records, administrative data, national censuses data, health surveys, and research studies. Knowledge translation literature is abundant with studies on information synthesis methods for producing the best available evidence. However, less attention has been paid to dissemination methods - how to make information available to policymakers. Policy guidance and policy briefs [2], and online evidence repositories [3] are methods of knowledge dissemination that have been used by leading health knowledge producers, including the World Health Organization and the Agency for Healthcare Research and Quality in the USA.

\section{Public Health Indicators}

Public health indicators are measures that reflect the status of specific aspects of public health and the health system. Public health indicators can be quantitative or qualitative. There are many types of indicators, including public health status (e.g., mortality rates, prevalence of diseases, the burden of diseases), health risks (e.g., obesity, smoking rates), healthcare programs' outcomes (cancer screening and vaccination rates), health system performance indicators (e.g., wait times in emergency departments, access rates), and health policy indicators (e.g., the cost of care). Organizations at international, national, provincial, and local levels select and use the types of indicators that best serve their planning and evaluation processes.

\section{Information Technology and Health Policymaking}

There is a scarcity of research on using information technology as a means for knowledge dissemination in health policymaking despite developments in information technology and the availability of tremendous amounts of data that can be used in health policymaking [4,5]. Many organizations, including the World Health Organization, the World Bank, and the Centers for Disease Control and Prevention publish health indicators databases online, such as healthcare outcomes, mortality and morbidity indicators, and health service utilization. These databases provide various tools to explore, sort, and extract the published information. Xu et al. [6] argue that these databases should be equipped with more sophisticated tools that enable users to analyze the indicators and explore associations and trends in data, rather than just listing the indicators. Alper et al. [7] suggest that health indicators are important not only for stakeholders' decision making but also for their continuous learning and understanding of the healthcare system; therefore, health indicators should be represented in ways that support these mental activities and goals such as decision making and learning.

\section{Interactive Visualization Tools}

Interactive visualization tools are external artifacts whose primary aim is to support and enhance users' exploratory and sense-making processes involving visually represented data [8-10] Visualizations encode abstract or concrete data (e.g., geographic, scientific, or health data) in a 
visual form. Visualizations can be static or interactive in nature. From the time John Snow used a map to reason about a cholera outbreak in 1850 to recent times, static visualizations have been used in public health. Though useful, static visualizations do not effectively support decisionmaking tasks. Visualizations can be made interactive. These tools use interactive visual representations to convey information and support decision-making tasks by allowing users to customize visualizations, and, in some cases, to perform analytical tasks [11].

Since policymaking requires public health stakeholders to reason with heterogeneous data, interactive visualization tools can play an important role. The effective and efficient use of data determines the extent to which stakeholders can sufficiently address policymaking issues. Therefore, tools that allow users to interact with information systematically can support decisionand policy-making activities.

Interactive visualization tools are used by decision makers in many domains, including business, engineering, and urban planning. These tools have a visual interface that enables humancomputer interaction and allows the user to organize and adjust the amount and representation of information. The interactive capabilities of these tools increase their "epistemic utility" and enhance cognitive task performance by increasing the user's information processing power $[10,12]$. Various visualization techniques can be used to represent data, including choropleth maps, heat maps, bubble charts, scatter plots and bar charts [13]. These techniques can use different visual marks (e.g., color, size, and shape) to encode different dimensions of information. Visualizations have increasingly been used to represent and communicate health data. However, according to a systematic review conducted by Carroll et al. [14], static graphics are still the main method for health data representation. There is scant literature on using visualization tools in healthcare [15]. Further research on how to develop domain-specific visualizations, user experience evaluation, and visualization techniques for heterogeneous health data is needed [16].

\section{Purpose of This Paper}

This paper reports an exploratory study whose purpose is threefold: 1) to design an interactive visualization tool that represents public health indicators and allows health policymakers to make sense of these indicators, 2) to examine the usability and utility of this tool, and 3) to investigate the benefits of this type of tool for policymakers.

In this paper, we only study the visualization of one type of health indicators: health equity indicators. Health equity, an overarching principle in the sustainable development goals [17], refers to "the absence of unfair and avoidable or remediable differences in health among population groups defined socially, economically, demographically or geographically" [18] (p. 12). Health equity indicators represent the disparities in health status among population groups.

\section{Study Method}

Interviewing prospective users is an effective research method in human-computer interaction research, which can be conducted in any of the product lifecycle phases [19]. The subjective feedback collected from prospective users enables researchers to understand the requirements, 
views, preferences and practices of users. Therefore, interviews might be as effective as ethnographic studies [19]. This method was used in our study.

We developed an online visualization tool that represents a set of health equity indicators in Canada. The study participants used and examined the tool. User experience was evaluated using the informal user experience evaluation method. This method involves the prospective users of a tool to use and play with it and their feedback to be collected through interviews. This method has been used to evaluate the usability and utility of computer programs and to identify design and functionality problems [20]. The method consists of three phases: Demonstration, exploration, and feedback interviews. These phases are summarized in Table 1. Participants went into these phases individually.

In the demonstration phase, the researchers present the visualization tool to the study participants. In our study, we presented the tool to some participants in person, and for other participants, who did not reside in our city, we created a video demonstration, in which we presented the tool using a brief tutorial.

In the exploration phase, study participants explored the tool freely and independently and for an unlimited time. They were not asked to perform any specific tasks using the tool. Once study participants felt that they were ready for an interview, the researchers conducted a feedback interview with each participant.

Using the interview method, we aimed to evaluate three aspects of the user experience: 1) the perceived ease of use, 2) the perceived usefulness of the tool, and 3) user satisfaction. We also wanted to understand users' needs and explore the tools' context of use. Therefore, we used a semi-structured interview with open-ended questions only. We had created an interview guide with a predefined set of themes before we started data collection, and we had updated this guide after each interview to reflect the emerging themes.

Table 1: The Informal User Experience Evaluation Method

\begin{tabular}{|l|l|l|l|l|}
\hline Phase & Name & Description & Location & Duration \\
\hline 1 & Demonstration & $\begin{array}{l}\text { The researcher will present } \\
\text { the tool to the participant. }\end{array}$ & $\begin{array}{l}\text { Participant } \\
\text { workplace }\end{array}$ & One hour \\
\hline 2 & Exploration & $\begin{array}{l}\text { A participant explores the tool } \\
\text { and performs any tasks on it } \\
\text { freely and without the } \\
\text { attendance of the researcher. }\end{array}$ & $\begin{array}{l}\text { Participant } \\
\text { workplace }\end{array}$ & $\begin{array}{l}\text { Open as per the } \\
\text { participant's } \\
\text { convenience }\end{array}$ \\
\hline 3 & $\begin{array}{l}\text { Feedback } \\
\text { Interview }\end{array}$ & $\begin{array}{l}\text { The researcher will interview } \\
\text { the participant and ask } \\
\text { him/her a set of open- ended } \\
\text { questions to evaluate the } \\
\text { perceived utility of the } \\
\text { visualization tool. }\end{array}$ & $\begin{array}{l}\text { Participant } \\
\text { workplace }\end{array}$ & $\begin{array}{l}\text { One session. } \\
\text { Expected duration } \\
\text { is two hours. }\end{array}$ \\
\hline
\end{tabular}




\section{Setting and Participants}

The informal user experience evaluation method, used in our study, requires that the visualization tool be evaluated by its prospective users, including health policymakers at different organizational levels, such as top-level managers with legal power to participate in decision making, and expert policymakers with technical and policy area expertise to analyze policy problems and suggest solutions. However, in the planning stage of the study, we anticipated that we would not be able to recruit top managers because of the time commitment required to participate in the study. Therefore, all the study participants were expert policymakers who work at various health system bodies in Ontario, such as local public health units, health research centers in hospitals and universities, local health integration networks, and community care centers. We used a purposeful sampling strategy (e.g., advertisement and snowballing) to target and recruit participants.

We had planned to recruit five to ten participants; we succeeded in recruiting seven participants. Table 2 provides a summary of participants' positions and education.

The Western University Research Ethics Board (protocol \#106967) approved this study.

Table 2: Participants' Positions and Education

\begin{tabular}{|c|c|c|c|}
\hline I & Alias Name & Professional Position & Education \\
\hline 1 & Participant\#1 & $\begin{array}{l}\text { Epidemiologist in a health } \\
\text { research centre }\end{array}$ & $\begin{array}{l}\text { M.Sc. \& P Ph.D. in } \\
\text { Epidemiology }\end{array}$ \\
\hline 2 & Participant\#2 & $\begin{array}{l}\text { Researcher in a health research } \\
\text { centre }\end{array}$ & R.N., Ph.D. \\
\hline 3 & Participant\#3 & Ph.D. student and researcher & R.N. \\
\hline 4 & Participant\#4 & $\begin{array}{l}\text { Program manager in a public } \\
\text { health unit }\end{array}$ & M.Sc. in Epidemiology \\
\hline 5 & Participant\#5 & $\begin{array}{l}\text { Assistant professor and } \\
\text { researcher }\end{array}$ & B.Sc.N., Ph.D. \\
\hline 6 & Participant\#6 & $\begin{array}{l}\text { Health records and business } \\
\text { analyst }\end{array}$ & M.Sc. in Health Science \\
\hline 7 & Participant\#7 & $\begin{array}{l}\text { Assistant professor } \\
\text { epidemiologist in a public } \\
\text { health unit }\end{array}$ & Ph.D. in Epidemiology \\
\hline
\end{tabular}

\section{Study Visualization Tool}

The tool used in this study is a website that includes a set of interactive visualizations. We used several visualization techniques, such as choropleth maps (Figure 1), scatter plots, heat maps, and bar charts. To support exploration of these visualizations, we used a number of interaction 
techniques, such as selecting, filtering, hovering, and zooming. We also used several visual marks, including shape, color, and size (Figure 2). Visualizations were developed using Tableau 9.2. To present the visualizations in an easy to navigate way, we visualized one of the conceptual frameworks of the social determinants of health (Figure 3), developed by Solar and Irwin [18], to represent and communicate the pathway of effects of the social determinants of health. This framework was intended to provide a theoretical lens through which policy action could be analyzed. The visualized health indicators and health outcomes were taken from research studies published in peer-reviewed journals.

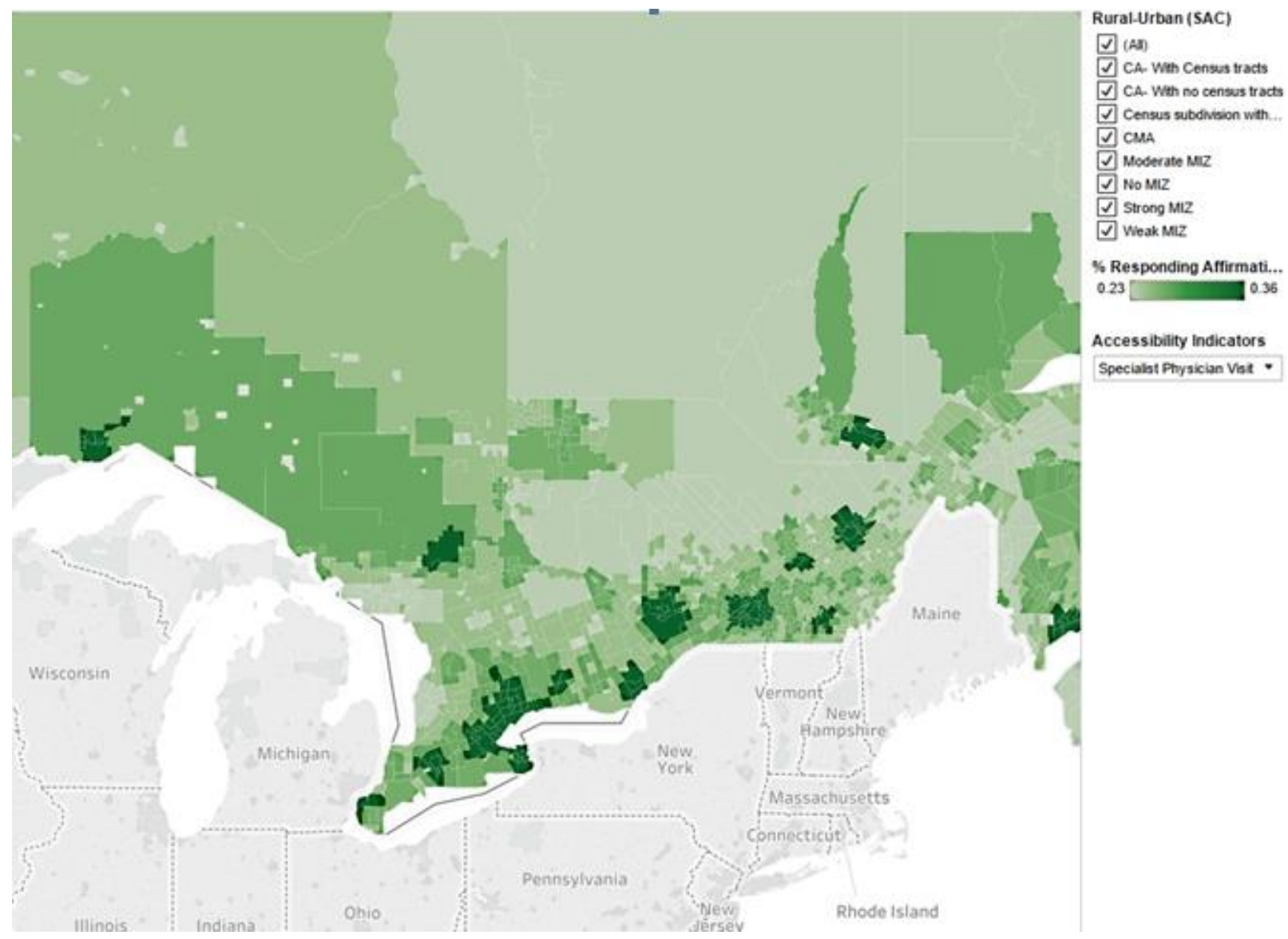

Figure 1: A choropleth map for a set of health indicators. These indicators represent access to healthcare services along the rural-urban continuum in Canada. The user can 1) select indicators from a list of indicators, 2) select rural and urban areas to see, and 3) zoom in and out on the data. 


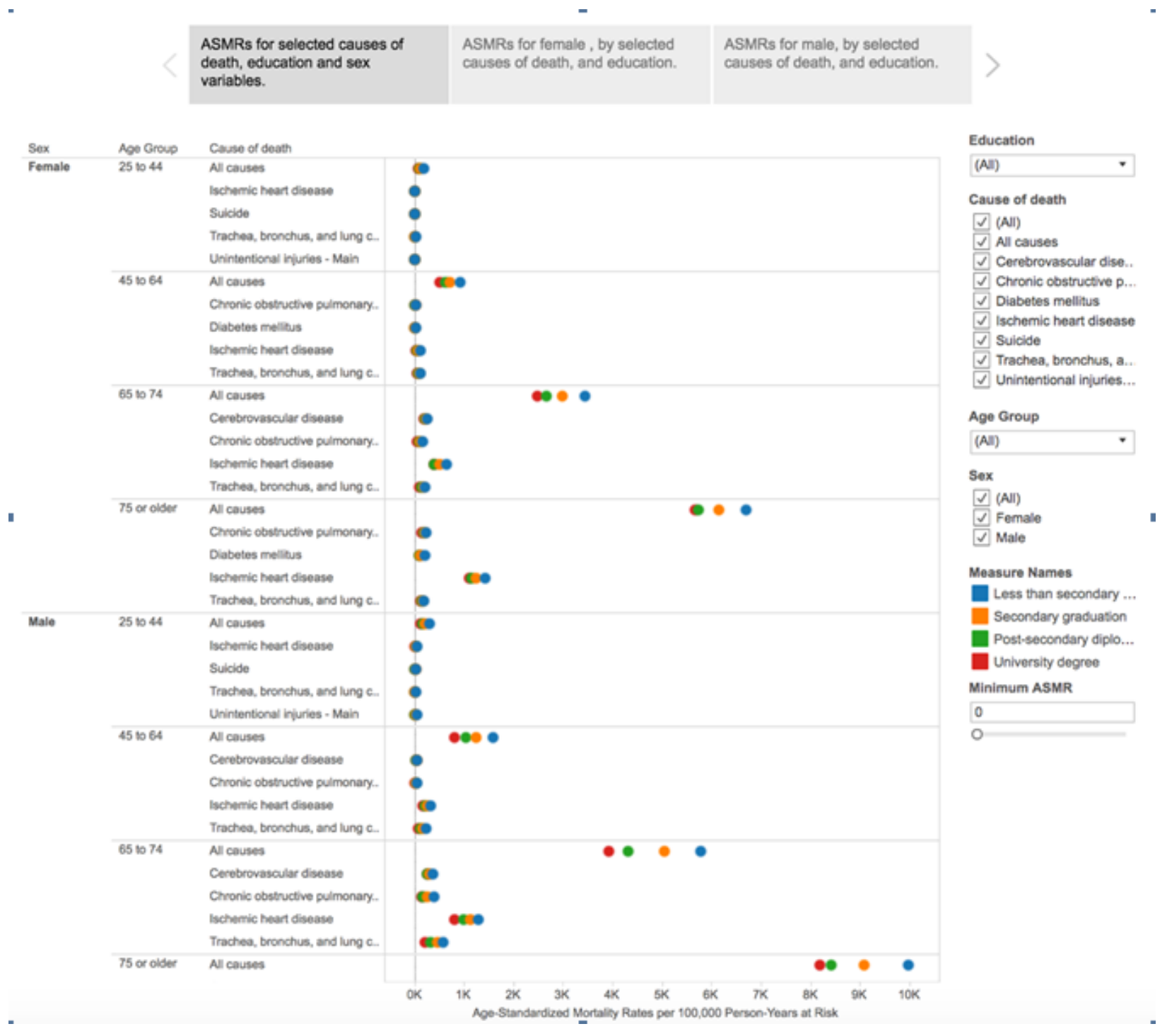

Figure 2: The cause-specific mortality by education in Canada.

\section{Data Analysis}

To qualitatively analyze the data, a data analysis software, QDA Miner Lite, was used. Data analysis in qualitative research is inductive and allows the emergence of themes from the data [21]. We used an a priori list, which we developed based on the core principles of software user experience and usability evaluation-namely, ease of use, user satisfaction, learnability, and effectiveness - to code the data. Despite using this list, we were still open to emerging themes, which we had not considered before the start of the study. Because our data analysis started in parallel with data collection, we were able to explore the emerging themes with other research participants and shed more light on those themes. 


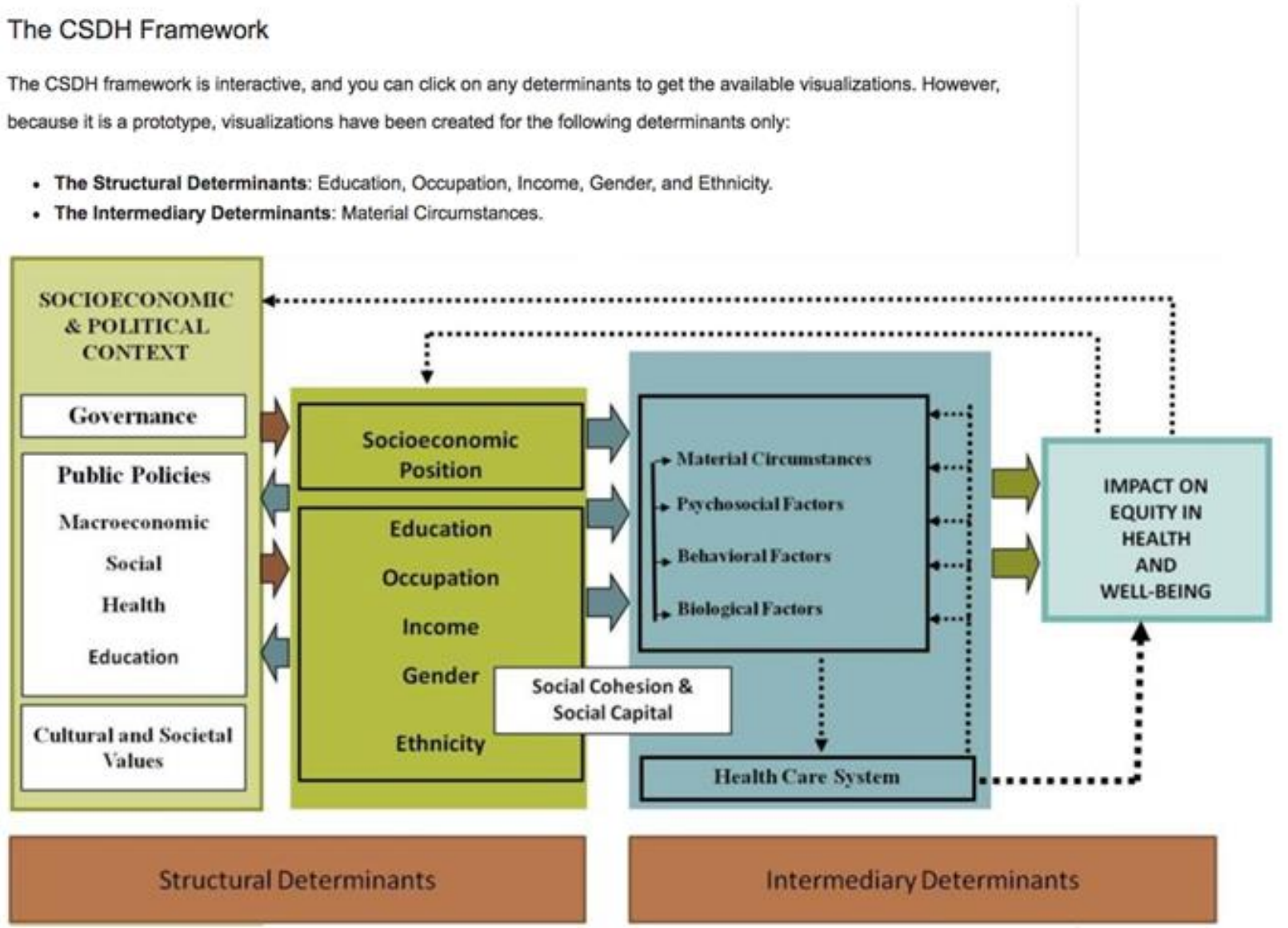

Figure 3: The conceptual framework for the social determinants of health as developed by Solar and Irwin [18]

\section{Results}

In this section, we present the themes that emerged, which represent the stories told by study participants at the interviews. Our role was to explicate these stories by objectively interpreting the content of these interviews.

\section{Usability, Utility, and User Experience Themes}

Participants were satisfied with the tool. The main reason for participant satisfaction was the interaction capabilities of the tool. These enable users to retrieve, filter and adjust the displayed information and were the main reason for participants' interest and satisfaction. Participants believed that the tool could be used for different purposes, such as communication, information dissemination, education, and decision support with regard to healthcare issues. Participant\#5, an assistant professor, believed that the tool is useful because it helps in presenting complex issues: "I think trying to make a more interactive dynamic way to explain very complex health and social phenomena is a worthy endeavor." 
Participants felt that the tool was easy to use. Some participants found that some visualizations were crowded, while others suggested using simpler visualizations and allowing users to add more layers of information to these visualizations. All participants preferred traditional charts (e.g., the bar charts) to more complex visualizations. Participant\#1, a data analyst and epidemiologist, felt that the simpler the visualizations, the better they are for the users:

"data visualization is trying to facilitate conceptualizing the data. But if you make it more complex, it loses the initial idea of visualizing the data, and If you are presenting it to people like healthcare professionals to help them better understand the relationships between the factors and the outcomes, I would say the simpler, the better."

As for the visual marks, participants felt that colors and tooltips were effective in representing the data. However, using too many visual marks could be overwhelming for the users. For some participants, interpretation of size as a visual mark was not easy. As for the visualization techniques, some participants felt that the choropleth map of health indicators was useful; however, other participants believed that map visualizations, in general, could lead to misinterpretation of the data. Filters were effective for controlling the visualizations. Participants found that visualizing the conceptual framework for the social determinants of health (Figure 3) is useful because it provides users with an overall view of the health determinants and the relationships among the different elements. However, despite the usefulness of having an overview of the health determinants, some participants felt that care should be exercised when including the visualizations of frameworks as these can impose on users a predefined structure which may not be universally acceptable to all users.

\section{Complexity of Knowledge Communication}

Participants identified a set of challenges that make knowledge communication in healthcare a complex task, including the inherent complexity of healthcare information and the diversity of audiences. These challenges can cause disconnection between knowledge producers and knowledge users. Participant\#4, an epidemiologist and a program manager at a health unit, described the difficulties that she faces at her work:

"As we roll out health status reports, we end up creating three different kinds of reports for three different kinds of users. When I first made the health and income inequalities report, I focused on the differences between PYLL (potential years of life lost) and mortality rates among income groups. However, I realized that no one understood what I was saying, and I tried to explain what PYLL was but people stared without a blink, and I knew I lost them.”

Most participants believed that visualization of information could facilitate or impede healthcare knowledge communication, depending on the usability of visualization tools. Additionally, some participants felt also that because of the inherent complexity of healthcare measurements and indicators, health policymakers should also have adequate training to be able to understand those indicators. Participant\#7, an assistant professor and epidemiologist in a health unit, described this issue: 
"I think that the education piece is what we need in order to elevate the decision makers' level of understanding for good epidemiologic analysis rather than simply accepting the results produced by epidemiologists."

Some of the participants talked about the possible misinterpretation of the represented information by laypeople.

Participant\#5, an assistant professor and a registered nurse, warned about misinterpreting the information:

"If you just give people the data without pushing them back to the theoretical understanding, there is a risk that they make their own conclusion. I think that this is the challenge of visualization because people may start asking different questions from what the data was meant for."

However, participants believed that the possible misinterpretation should not lead to not visualizing healthcare information; rather, we need to add more information about the visualized studies to help users fully understand the visualized data.

The validity and credibility of the visualized data were very important to study participants; they emphasized the need to include more information about the source studies, including the research questions, context, conclusions and the limitations of those studies. Participants also suggested establishing quality criteria to select the studies that will be included and visualized in the tool.

\section{Health Policymakers' Needs}

Participants stated that health policymakers do not have enough time for learning and using decision support tools such as our visualization tool. Participants also believed that policymakers prefer health reports that are summarized and include simple graphics. Participant\#7, an assistant professor and epidemiologist, did not think that the tool can be easily accepted by policymakers:

"I found your visualization very understandable, but I don't think that it is easily transferable to decision makers without a lot more background for them. However, my personal experience is that many decision makers don't really want to spend the time to understand these things. "

Participants stated that data and decision support tools such as information visualization tools play a secondary role in health policymaking compared to other factors that have a bigger impact, including public and media pressure, financial constraints, and political priorities. Participant\#5, an assistant professor, believed that information about healthcare issues is less important than the media pressure for action:

"The way that data is presented is less important than a couple of newspaper articles that make the government look really bad about the issue, so I think the number one factor is that policymakers have a sense that this is a priority issue, and probably the secondary thing is that they can understand the best way to approach the issue, and that is when they would get into how the data is being presented." 


\section{Discussion}

Our study focused on representing health equity indicators using interactive visualization tools and the benefits of these tools for health policymaking. From an information technology perspective, there are three related elements that should be analyzed: the tool, the users of the tool, and the visualized data.

\section{The Tool}

Our study shows that using interactive visualization tools to represent health indicators has advantages over traditional representation techniques that do not allow users to interact with the information. Despite this, a systematic review conducted by Carroll et al. [14]analyzing the information needs of public health professionals highlights the fact that while interactive visualization boosts users' capabilities and enhances their experience, health indicator visualization still predominantly depends on static graphics.

To gain the full benefits of interactive visualizations tools, the developers of those tools should follow the user-centered design approach, which includes understanding users' needs, focusing on their task and work processes, iterative design, and continuous user experience testing [22]. However, many visualization tools are designed to impress users rather than help them perform their work tasks effectively and efficiently. We believe that healthcare informatics tools for policymakers should be designed to fit their tasks and needs.

\section{The Users of The Tool}

In our interviews with the participants, we find that there are many knowledge gaps on healthcare data communication and more research is required to explore this topic.

\section{Policymakers}

There are increasing calls to different sectors, such as business, healthcare, and government, to adopt and use data analytics and visualization software in policymaking. The study in this paper re-emphasizes the fact that health policymaking is a complex process; data is only one element among others needed in this complex process. Therefore, we believe that researchers and healthcare organizations should conduct a strategic evaluation to assess the usability of these tools before using them. Such evaluation should take into consideration the cost and the traditional usability metrics, including ease of use, learnability, and efficiency; it should also look at the policymaking process and the numerous factors that affect this process, including information needs, political factors, financial constraints, and stakeholders' values and beliefs. This evaluation will help healthcare organizations in estimating the return on investment that they will have, and whether they should invest in these tools.

\section{Laypeople and misinterpretation of health outcomes}

Information misinterpretation occurs when users of the information ignore or do not know why, where, and how it was produced, or where and how it can be used [23]. Our tool presents 
research studies that show the association between health disparities and the socioeconomic status of population groups. However, the tool only shows the results of the studies (i.e., health outcomes); it leaves out other important parts of those studies, including the context and limitations of each study. This omission may lead to misinterpretation. Misinterpretation can also happen when users of the tool lack enough understanding of the health issues that are represented in the tool. We believe that the complexity of association between social determinants of health and health outcomes, and the multilevel pathway of effects are always ignored, lost, or forgotten whenever those outcomes are summarized (i.e., in blogs), reported in the media, or represented visually.

We propose here the following guidelines for the publishers of this data to reduce the possibility of misinterpretation of healthcare indicators by laypeople:

1- Cite and provide a list of the studies from which the data was obtained.

2- Mention the context of those studies.

3- Mention the limitation of those studies.

4- As we have done in our tool, provide a conceptual framework that represents the association between the several independent factors on the one hand, and the health outcomes - the dependent factors - on the other hand. This framework helps the users to understand the phenomena that you are representing.

5- Remind the users of the data that the social determinants of health affect people's health through complex and multilevel pathways, leading to different healthcare outcomes that can't be attributed to any single determinant alone.

\section{The Visualized Data}

Knowledge translation literature has traditionally focused on the needs of information users, including healthcare providers and policymakers, and on the types and content of knowledge products that best fit those needs. However, less attention has been paid to the difficulty of producing these products, as suggested in this paper. This shortcoming is partly due to the inherent complexity of some healthcare indicators, requiring users to have a certain level of understanding of statistical and epidemiological data. Some healthcare providers and most policymakers lack such understanding, forcing knowledge producers (i.e., researchers and epidemiologists) to exert extra effort to simplify health reports and replace certain indicators with easy-to-understand ones. This practice makes those reports less comprehensive and more generalized and threatens information quality. Even when information technology tools are used to communicate and represent health reports and indicators, these reports should be simple [14]. Therefore, more research is required to explore this issue and design innovative methods to communicate healthcare information and outcome indicators.

\section{Conclusions}

Interactive visualization tools can be used to represent and communicate public health indicators. However, in health policymaking, the ultimate value of interactive visualization tools is influenced by diverse factors, including the complexity of health indicators, the usability of the tools, and policymakers' needs, capabilities and priorities. These factors should be taken into 
consideration before investing in interactive visualization software. Effective communication of health indicators requires more research to model the complexity of health indicators, and the needs of knowledge producers and users.

Finally, our study shows that interactive visualization tools have great potential to address the needs of healthcare knowledge management and policymaking. However, for this potential to be actualized, more research is required to develop standard metrics to assess the usability and utility of these tools in supporting healthcare policymaking in different contexts.

\section{Limitations of This Study}

Usability of interactive visualization tools in health policymaking and health communication are rarely discussed in the literature. While our study sheds light on how to design these tools for these purposes, since it is an exploratory study, it has some limitations.

Firstly, as we described in the method section, interviewing prospective users was used in this study. This method is a legitimate qualitative method in human-computer interaction research. We used purposeful sampling to recruit study participants. A small sample size was used--one that is sufficient for exploratory such studies. Our goal was to recruit participants in various policymaking positions, but ended up recruiting experts in decision-support positions, as the former were difficult to recruit.

Secondly, we tried to create simple and familiar visualizations to facilitate the evaluation of our visualization tool by study participants. However, it is possible that the tool's design and features impacted participants' opinions.

Finally, our research method and sampling strategy have an impact on the generalizability of our findings. As is the case with all qualitative research, we can only claim "tentative application" of our findings because these findings are context-dependent [24]. It is the responsibility of other researchers, wishing to use these findings, to verify their applicability in similar or different contexts.

We recommend that future research studies use field deployment methods, which could provide deeper insights into the users' needs in the real context of use, and the impact of this context on the user experience and usefulness of the information technology artifacts. When appropriate human and financial resources are available for non-exploratory studies, field deployment methods allow researchers to use a variety of quantitative and qualitative methods to evaluate the usability of such artifacts.

\section{Declaration of Conflicting Interests}

The authors declare that there are no potential conflicts of interest with respect to the research, authorship, and/or publication of this article.

\section{References}

1. Buse K, Mays N, Walt G. Making health policy. McGraw-Hill Education (UK); 2012. 
2. Bosch-Capblanch X. Handbook for supporting the development of health system guidance. Basel Swiss Cent Int Heal. 2011

3. Dobbins M, Hanna SE, Ciliska D, Manske S, Cameron R, et al. 2009. A randomized controlled trial evaluating the impact of knowledge translation and exchange strategies. Implement Sci. 4(1), 61. PubMed https://doi.org/10.1186/1748-5908-4-61

4. Bassi J, Lau F. 2013. Measuring value for money: a scoping review on economic evaluation of health information systems. J Am Med Inform Assoc. 20(4), 792-801. PubMed https://doi.org/10.1136/amiajnl-2012-001422

5. Quinn E, Huckel-Schneider C, Campbell D, Seale H, Milat AJ. 2014. How can knowledge exchange portals assist in knowledge management for evidence-informed decision making in public health? BMC Public Health. 14(1), 443. PubMed https://doi.org/10.1186/1471-2458-14-443

6. Xu S, Jewell B, Steed C, Schryver J. A new collaborative tool for visually understanding national health indicators. In: Proceedings of the International Conference on Applied Human Factors and Ergonomics. 2012. p. 91-100.

7. Saunders R, Sanders J, Alper J, eds. Core measurement needs for better care, better health, and lower costs: counting what counts: workshop summary. National Academies Press; 2013.

8. Card SK, Mackinlay JD, Shneiderman B. Readings in information visualization: using vision to think. Morgan Kaufmann; 1999.

9. Robert S. Information Visualization-Design for Interaction. UK Pearson Educ Ltd. 2007

10. Sedig K, Parsons P. 2013. Interaction design for complex cognitive activities with visual representations: A pattern-based approach. AIS Trans Human-Computer Interact. 5(2), 84-133.

11. Ola O, Buchel O, Sedig K. 2016. Exploring the Spread of Zika: Using Interactive Visualizations to Control Vector-Borne Diseases. Int J Dis Control Contain Sustain. 1(1), 47-68. https://doi.org/10.4018/IJDCCS.2016010104

12. Fekete J-D, Van Wijk J, Stasko J, North C. The value of information visualization. In: Information visualization. Springer; 2008. p. 1-18.

13. Sedig K, Parsons P. 2016. Design of Visualizations for Human-Information Interaction: A Pattern-Based Framework. Synth Lect Vis. 4(1), 1-185. https://doi.org/10.2200/S00685ED1V01Y201512VIS005

14. Carroll LN, Au AP, Detwiler LT, Fu T, Painter IS, et al. 2014. Visualization and analytics tools for infectious disease epidemiology: a systematic review. J Biomed Inform. 51, 287-98. PubMed https://doi.org/10.1016/j.jbi.2014.04.006 
15. Steenstra D, Erkoyuncu JA. Using Visualisation for Disruptive Innovation in Healthcare. In: Virtual, Augmented Reality and Serious Games for Healthcare 1. Springer; 2014. p. 111-42.

16. Liu S, Cui W, Wu Y, Liu M. 2014. A survey on information visualization: recent advances and challenges. Vis Comput. 30(12), 1373-93. https://doi.org/10.1007/s00371-013-0892-3

17. Organization WH. World Health Statistics 2016: Monitoring Health for the SDGs Sustainable Development Goals. World Health Organization; 2016.

18. Solar O, Irwin A. A conceptual framework for action on the social determinants of health. World Health Organization; 2010.

19. Lazar J, Feng JH, Hochheiser H. Research methods in human-computer interaction. Morgan Kaufmann; 2010.

20. Lam H, Bertini E, Isenberg P, Plaisant C, Carpendale S. 2012. Empirical studies in information visualization: Seven scenarios. IEEE Trans Vis Comput Graph. 18(9), 1520-36. PubMed https://doi.org/10.1109/TVCG.2011.279

21. Creswell JW. Research design: Qualitative, quantitative, and mixed methods approaches. Sage publications; 2013.

22. Ritter FE, Baxter GD, Churchill EF. Foundations for designing user-centered systems: what system designers need to know about people. Springer Science \& Business Media; 2014.

23. Eppler MJ. A generic framework for information quality in knowledge-intensive processes. In: Proc sixth international conference on information quality. 2001. p. 329-46.

24. Guba EG, Lincoln YS. Competing paradigms in qualitative research. In: Denzin NK, Lincoln YS, editors. Handbook of qualitative research. Sage, Thousand Oaks, CA; 1994. p. $105-17$. 